Erildo Vicente Müller

Eliane Aparecida Campesatto

Fabiana Bucholdz Teixeira Alves

Giovani Marino Favero

Rafael Gomes Ditterich

Organizadores

\title{
COVID-19 \\ ORIENTAÇÕES PARA GESTANTES E PUÉRPERAS
}
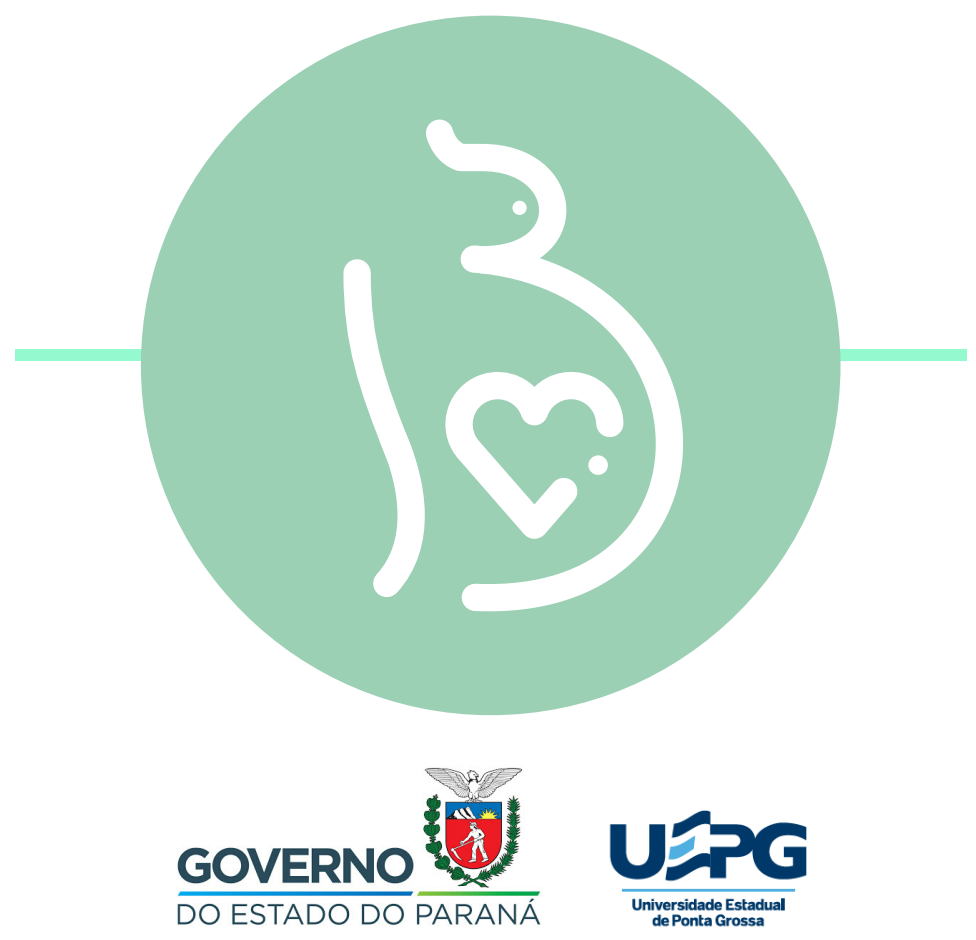


\title{
GOVERNO DO ESTADO DO PARANÁ
}

\section{Governador}

Carlos Massa Ratinho Júnior

\section{Vice Governador}

Darci Piana

\section{Superintendência Geral de Ciência, Tecnologia e Ensino Superior \\ Aldo Nelson Bona}

\section{UNIVERSIDADE ESTADUAL DE PONTA GROSSA}

\author{
Reitor \\ Miguel Sanches Neto \\ Vice Reitor \\ Everson Augusto Krum
}

\section{Pró-Reitoria de Extensão e Assuntos Culturais} Clóris Regina Blanski Grden
Programa de Pós-Graduação em Ciências da Saúde Dionízia Xavier Scomparim

\section{Programa de Residência Multiprofissional em Saúde} Luciane Patrícia Andreani Cabral

\author{
Colaboradores \\ Bruno Diniz Batista \\ Fabiana Bucholdz Teixeira Alves \\ Jessica Galvan \\ Larissa Guil \\ Laryssa de Col Dalazoana Baier \\ Luiz Ricardo Marafigo Zander \\ Mariana Xavier Borsoi \\ Matheus Pelissari Migliorini \\ Mittalys Wuana Pilatti Andrade e Silva
}




$$
\begin{gathered}
\text { COVID-19 } \\
\text { Orientações } \\
\text { para gestantes } \\
\text { e puérperas }
\end{gathered}
$$




\title{
PROEX UEPG
}

\section{Equipe Editorial}

\section{Revisão de língua portuguesa}

Fábia Mariela De Biasi

\author{
Arte \\ Larissa Guil \\ Diagramação \\ Andressa Marcondes
}

Apoio

Editora UEPG

C873

COVID-19: orientações para gestantes e puérperas [livro eletrônico]/ Erildo Vicente Müller et al. (org.). Ponta Grossa: UEPG/PROEX, 2020.

39 p.; E-book PDF

ISBN: 978-65-86967-01-2

DOI: 10.5212/86967-01-2

1. Corona vírus. 2. COVID-19- sintomas. 3. Gravidez. 4. Puerpério. I. Müller, Erildo Vicente (Org.). II. Campesatto, Eliane Aparecida (Org.). III. Favero, Giovani Marino (Org.). IV. Alves, Fabiana Bucholdz Teixeira (Org.). V. Ditterich, Rafael Gomes (Org.). V. T.

CDD: 618.2

Ficha Catalográfica elaborada por Maria Luzia F. B. dos Santos - CRB9/986 


\section{APRESENTAÇÃO}

Prezado Leitor:

Diante da emergência da pandemia do novo coronavírus, com tantas informações circulando via web e várias notícias que afligem e assustam a população, o Grupo de Estudos em Epidemiologia e Cuidados em Saúde e o Grupo de Enfrentamento da Covid-19 reuniram informações baseadas na melhor evidência científica disponível no momento para as gestantes e puérperas, traduzindo-as de maneira acessível à população.

Esperamos, com este material, contribuir para o esclarecimento e a prevenção da Covid-19, pois esse é nosso papel enquanto cientistas e acadêmicos da Universidade Estadual de Ponta Grossa: fornecer subsídios para que a pandemia possa ser enfrentada de maneira consciente e com informação de qualidade.

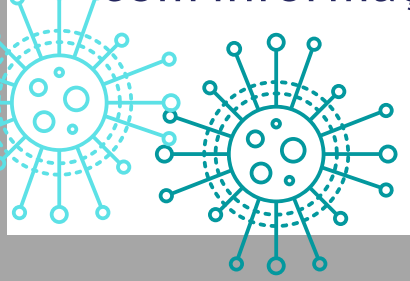

Boa leitura! 


\title{
COVID-19 O QUE É?
}

Doença causada por um vírus da SARS-COV-2 família do coronavírus

\section{O QUE CAUSA?}

Uma infecção que afeta principalmente o sistema respiratório

\section{CUIDADO!}

PODE SER

CONFUNDIDA COM

\author{
GRIPE OU \\ RESFRIADO
}




\section{POR QUE A COVID-19 É UMA PANDEMIA?}

ESTÁ PRESENTE EM TODOS OS CONTINENTES

\section{EM 23 DE ABRIL DE 2020}

2.647.512 CASOS CONFIRMADOS

184.643 MORTES

Organização Mundial da Saúde (OMS)

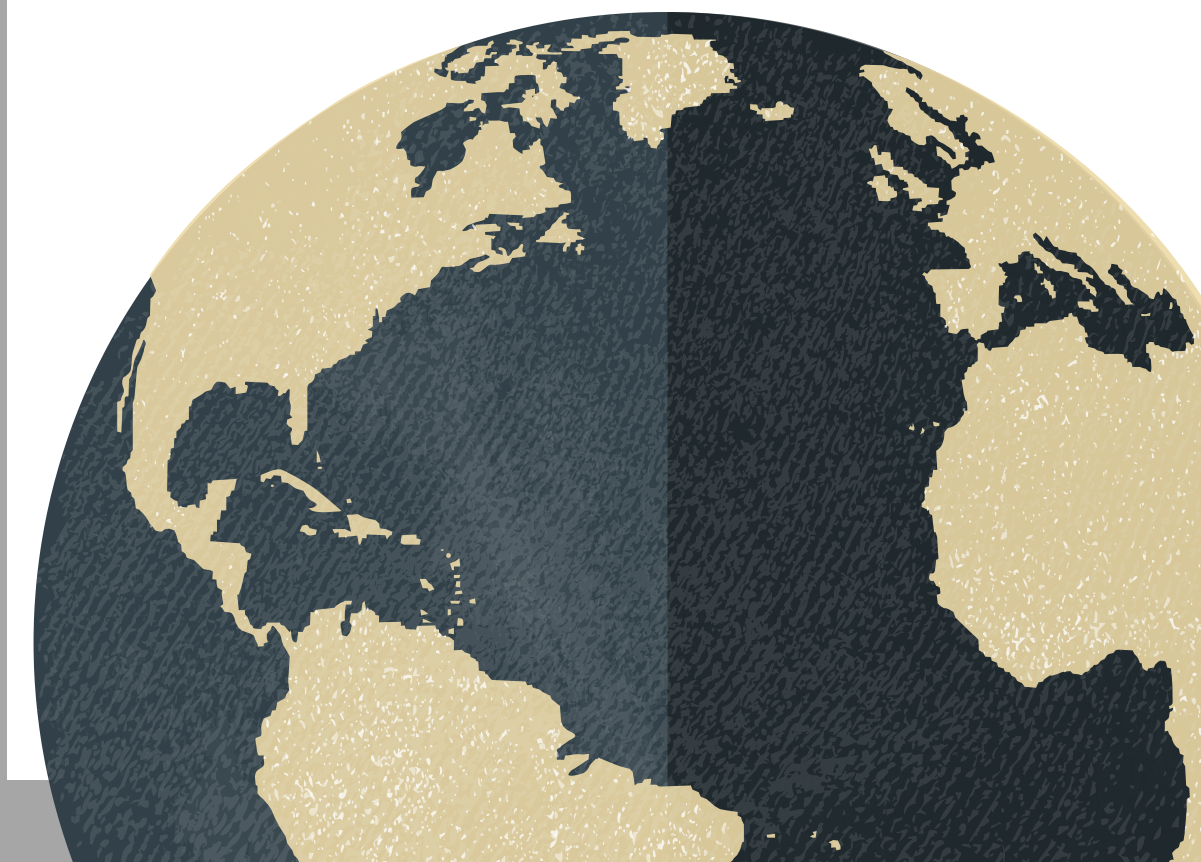




\section{O VÍRUS ESTÁ}

\section{PRESENTE NA SALIVA}

\section{e também nas}

gotículas do nariz ou

da boca, que se

espalham no ar e em

superfícies se uma pessoa infectada tossir ou espirrar 


\section{SINAIS E SINTOMAS}

PACIENTES PODEM SER CLASSIFICADOS

EM 3 CATEGORIAS CLIINICAS

\section{ASSINTOMÁTICOS}

\section{FORMA LEVE}

FORMA GRAVE
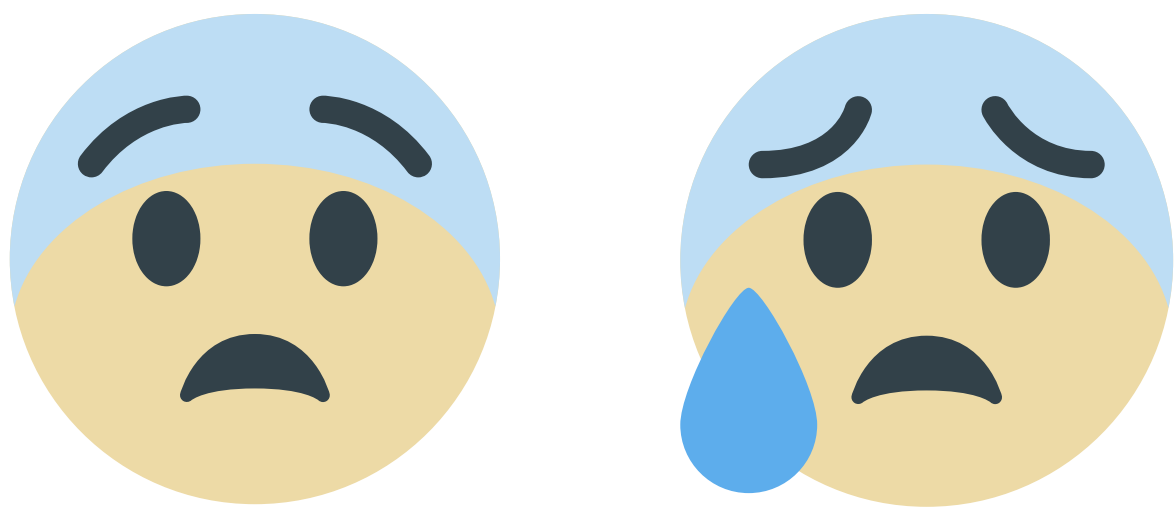


\section{ASSINTOMÁTICOS}

INDIVÍDUOS INFECTADOS QUE NÃO MANIFESTAM A DOENÇA

\section{TRANSMITEM O VÍRUS PARA A COMUNIDADE}

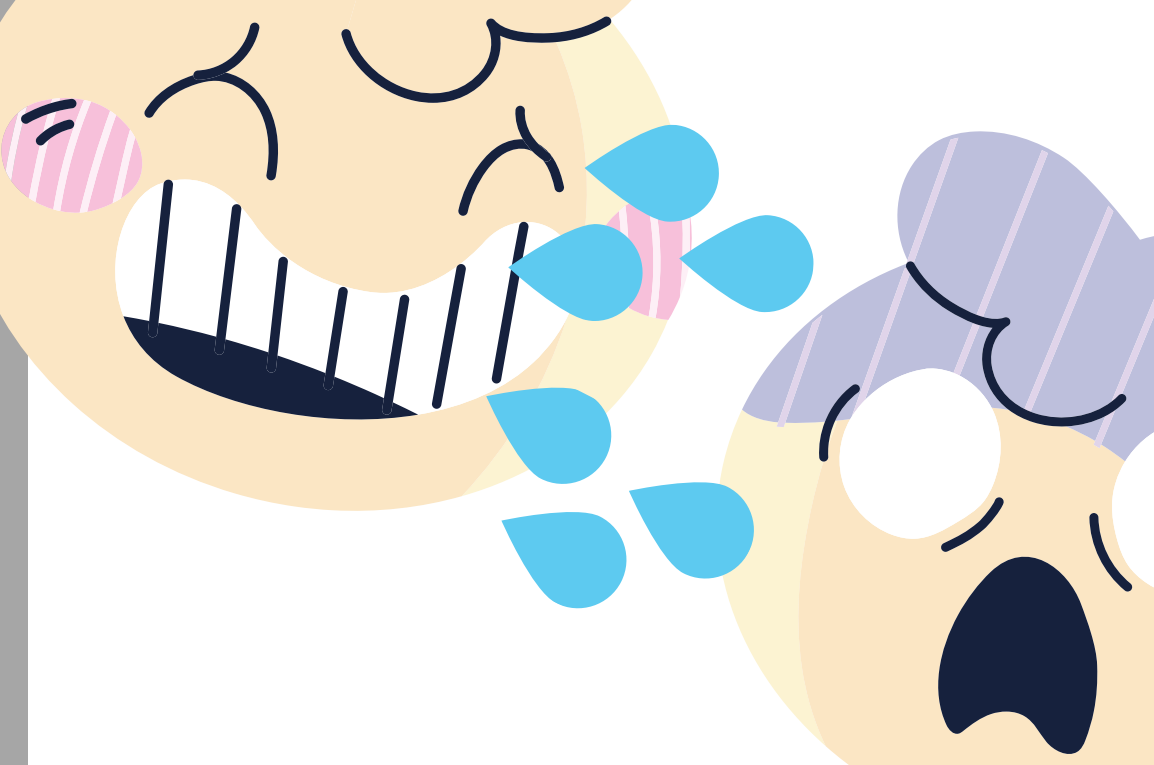




\section{FORMA LEVE}

\section{FEBRE, CANSAÇO, TOSSE SECA, CONGESTÃO E CORRIMENTO NASAL, DOR DE GARGANTA, DIARREIA}
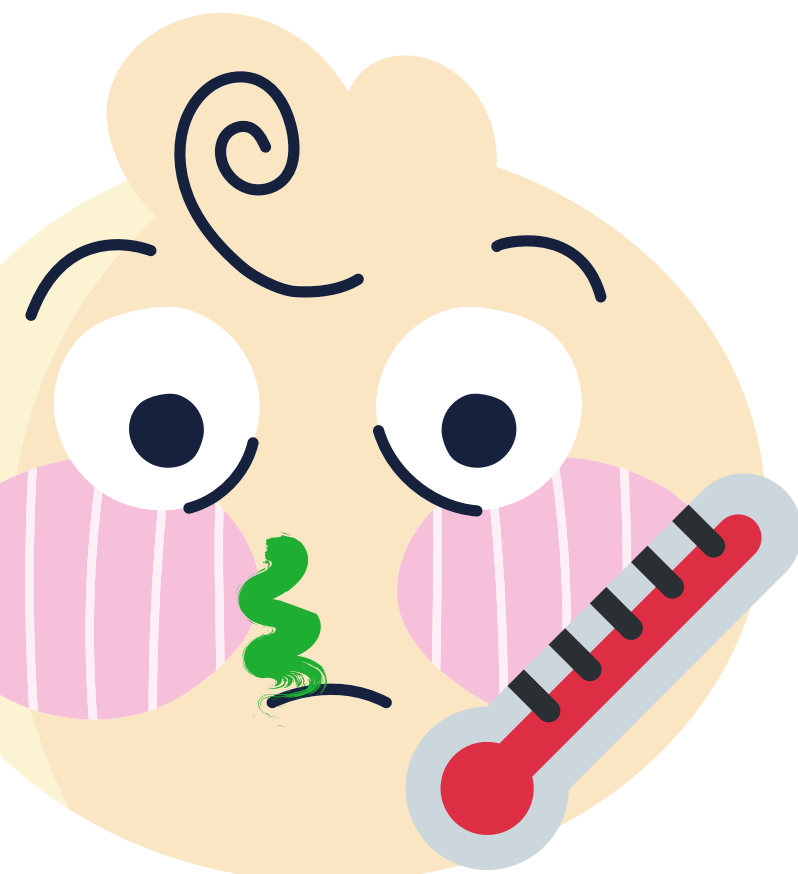


\title{
FORMA GRAVE
}

FEBRE, CANSAÇO, TOSSE SECA, CONGESTÃO E CORRIMENTO

NASAL, DOR DE GARGANTA, DIARREIA

\author{
$+$ \\ DIFICULDADE PARA RESPIRAR
}

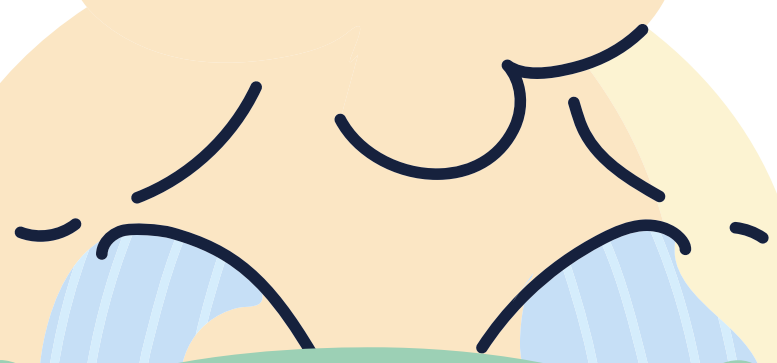




\section{QUANDO DEVO PROCURAR \\ ATENDIMENTO MÉDICO? \\ Quando tiver:}

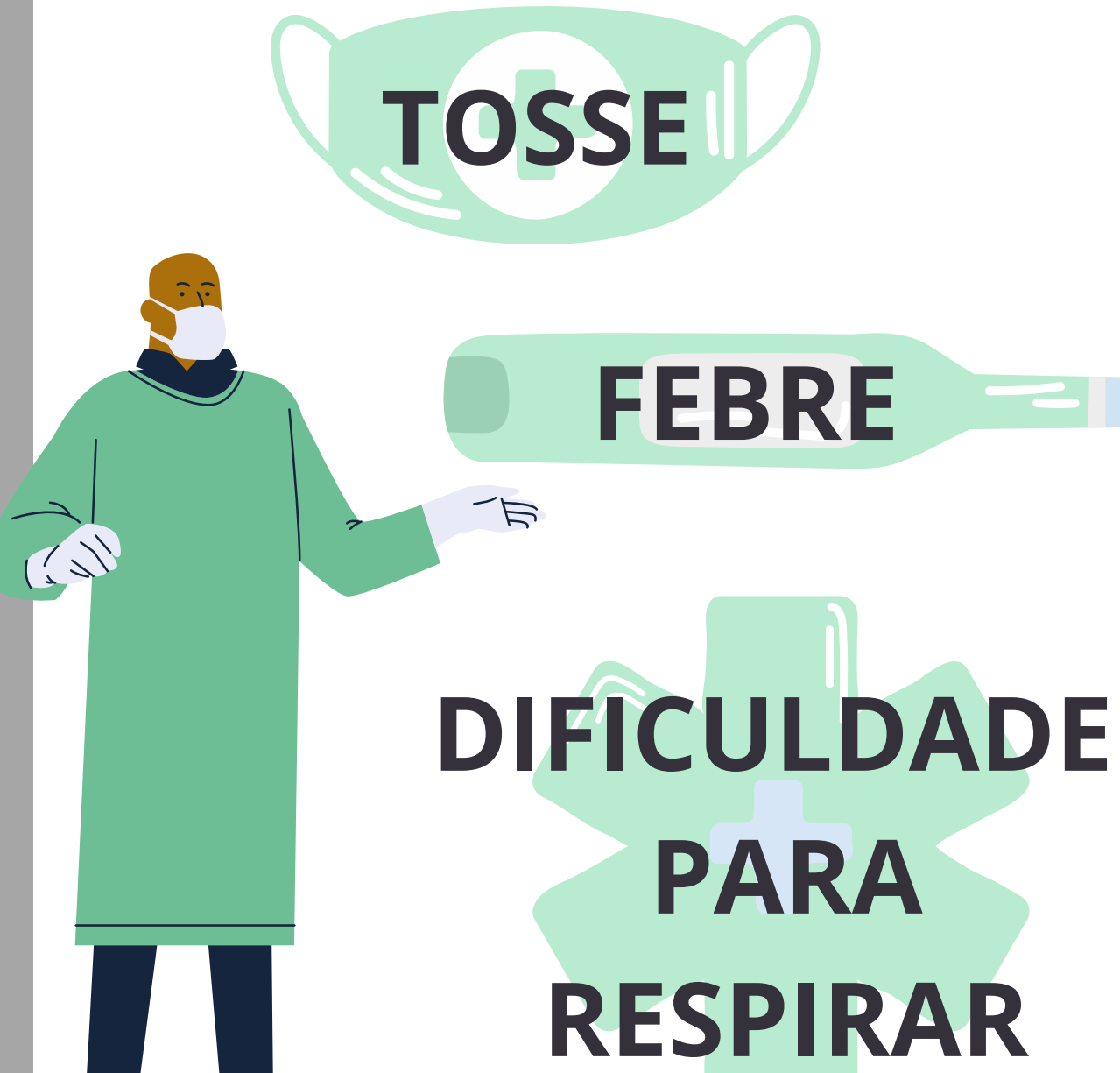


Gestantes

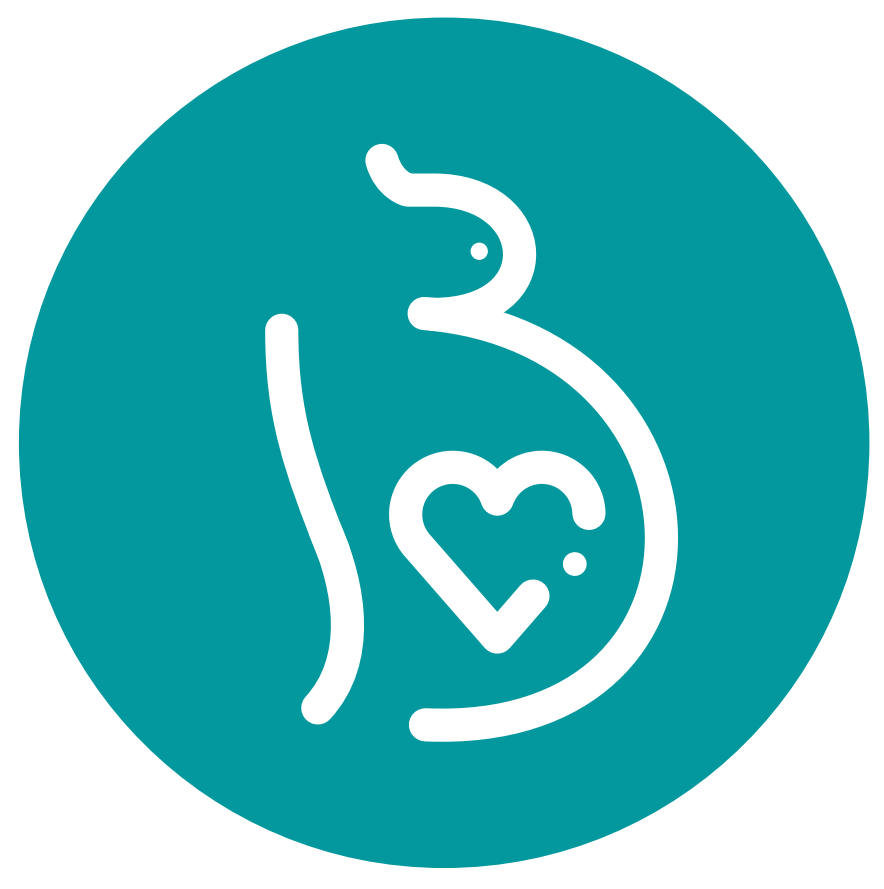




\section{GESTANTES NÃO TÊM DEMONSTRADO}

MAIOR SUSCETIBILIDADE À INFECÇ̃̃O
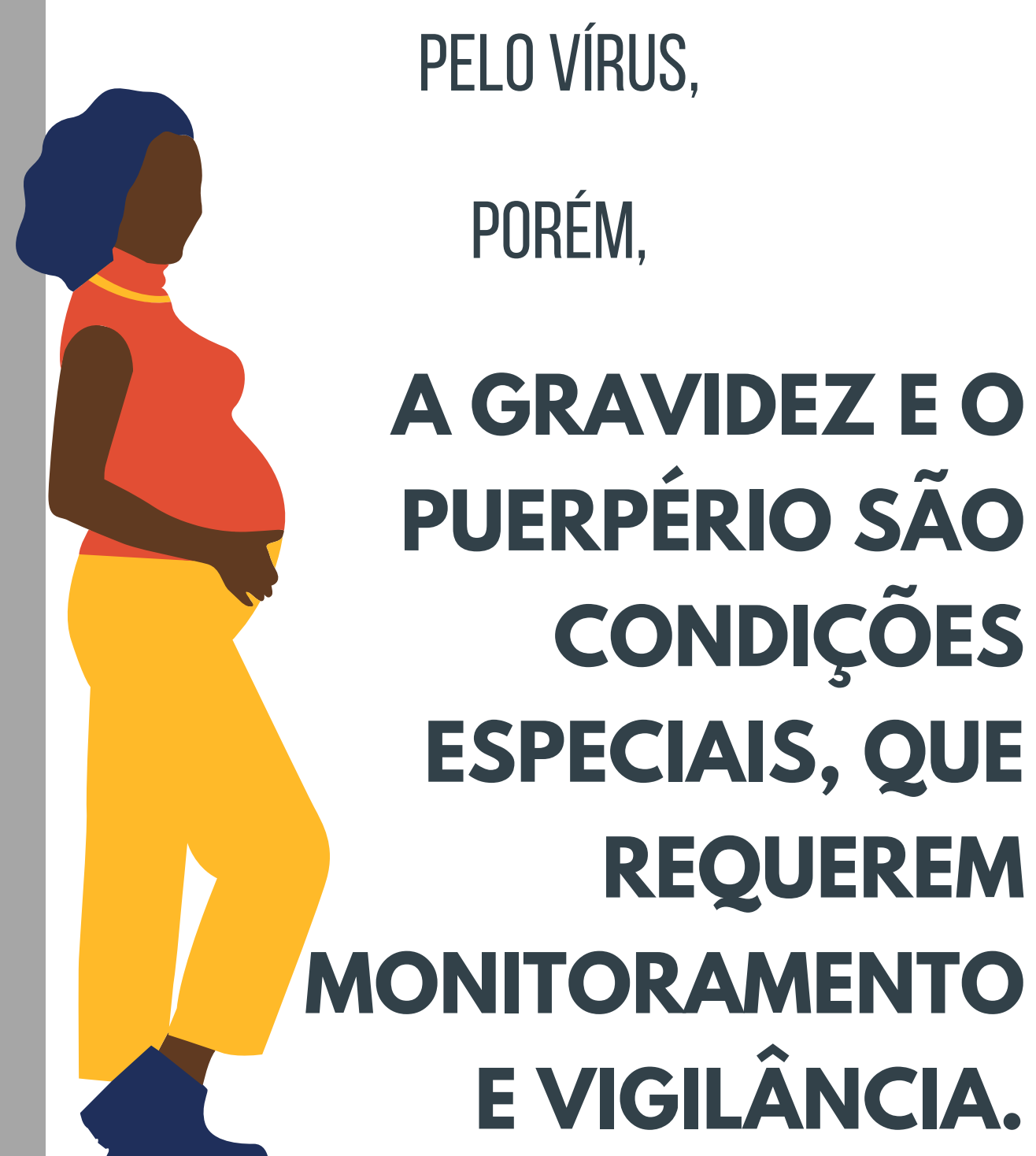
Os atendimentos a essa população não foram interrompidos e,

segundo a Secretaria da Saúde do Paraná,

devem permanecer em ambientes seguros,
devidamente
organizados e
higienizados 


\section{Mulheres grávidas}

apresentam risco maior?

\section{O Ministério da Saúde}

\section{afirma que sim}

Ainda não há estudos conclusivos que comprovem maior perigo da Covid-19 para grávidas e puérperas,

\section{A INCLUSÃO NO GRUPO \\ DE RISCO CONSIDEROU A}

AÇÃO DE OUTRAS VIROSES

JÁ CONHECIDAS E

ESTUDADAS 


\section{Durante o período}

gestacional, o sistema imunológico da mulher está alterado,

$$
\text { por essa razão, }
$$

HÁ MAIOR CHANCE DE

$$
\text { INFECÇÃO }
$$

RESPIRATÓRIA 


\section{O vírus pode ser \\ transmitido ao bebê?}

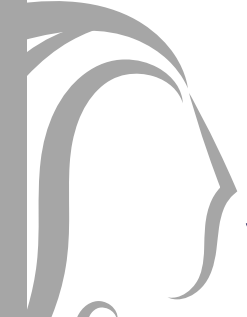

Não há evidências de que o vírus possa ser transmitido ao feto durante a gravidez ou o parto

A MAIORIA DOS BEBÊS E CRIANÇAS APRESENTA APENAS FORMAS LEVES DE COVID-19

Mesmo assim, é FUNDAMENTAL seguir as orientações dos órgãos de saúde 
Se não há evidências até

o momento, por que

gestantes e puérperas

são consideradas grupo de risco?

O principal motivo é a cautela, por isso

devemos 


\section{SE EU FICAR DOENTE, \\ O QUE DEVO FAZER?}

\section{Se apresentar:}

\section{TOSSE}
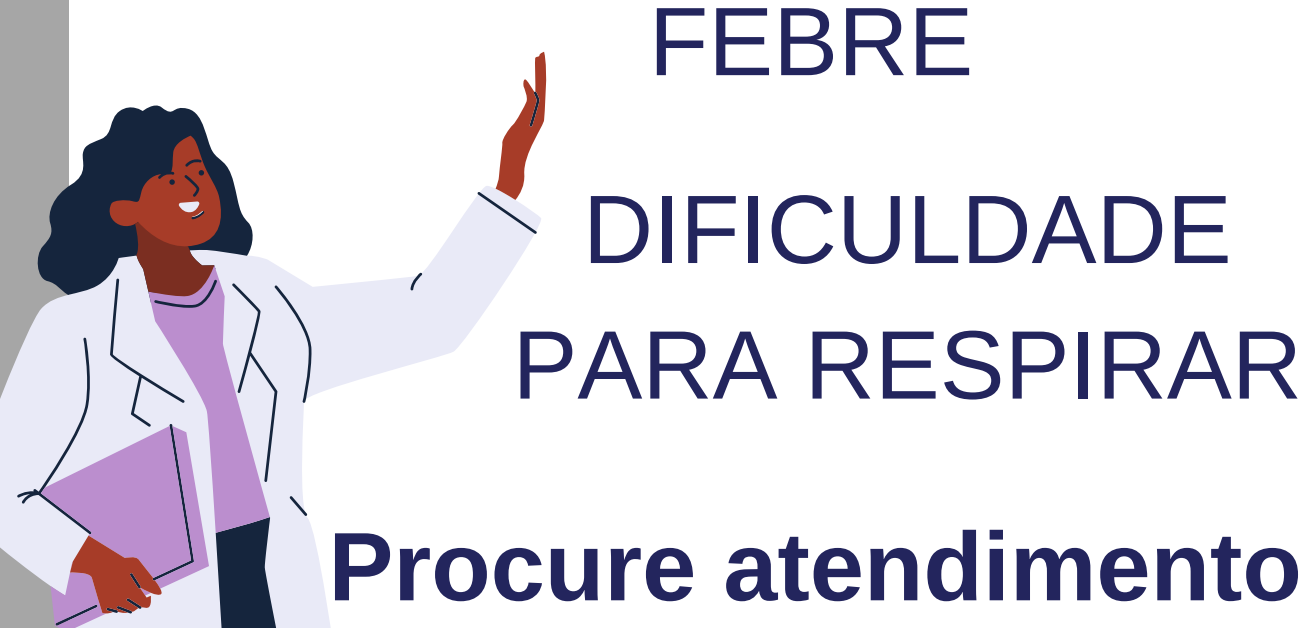

Procure atendimento médico

- médic

MENCIONE QUE

ESTÁ GRÁVIDA 


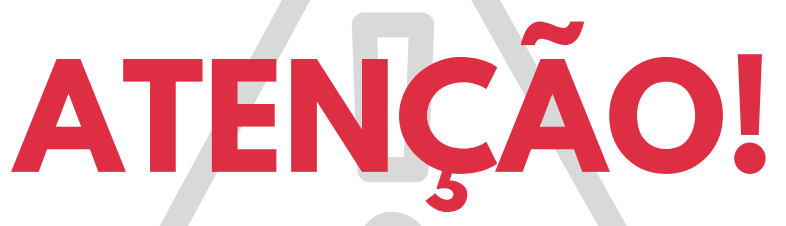

\section{ENTRE EM CONTATO COM A}

\section{EQUIPE RESPONSÁVEL PELO}

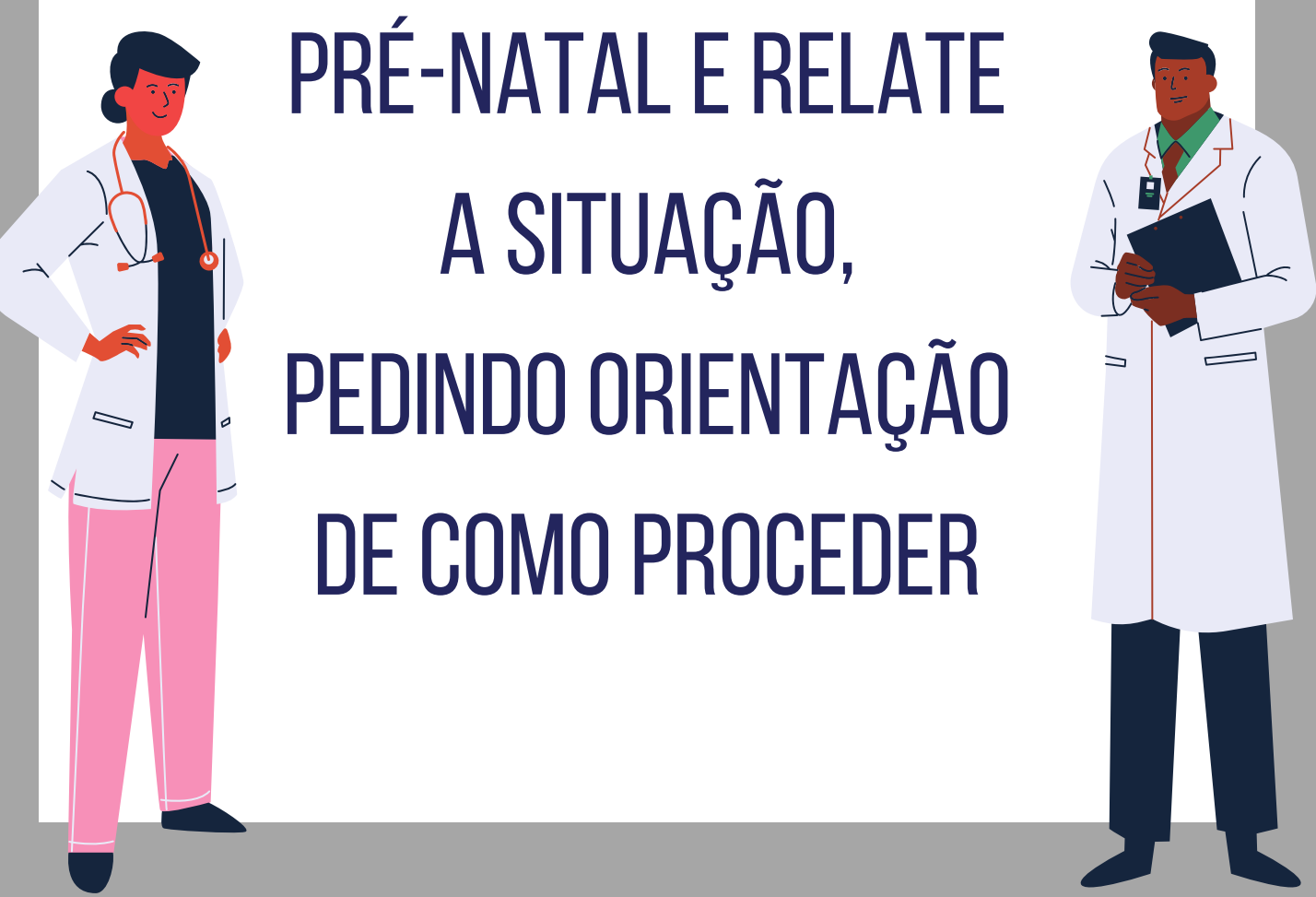




\section{O que fozer}

\section{como grávida?}

Mantenha-se

informada sobre a

Covid-19 por meio de fontes confiáveis
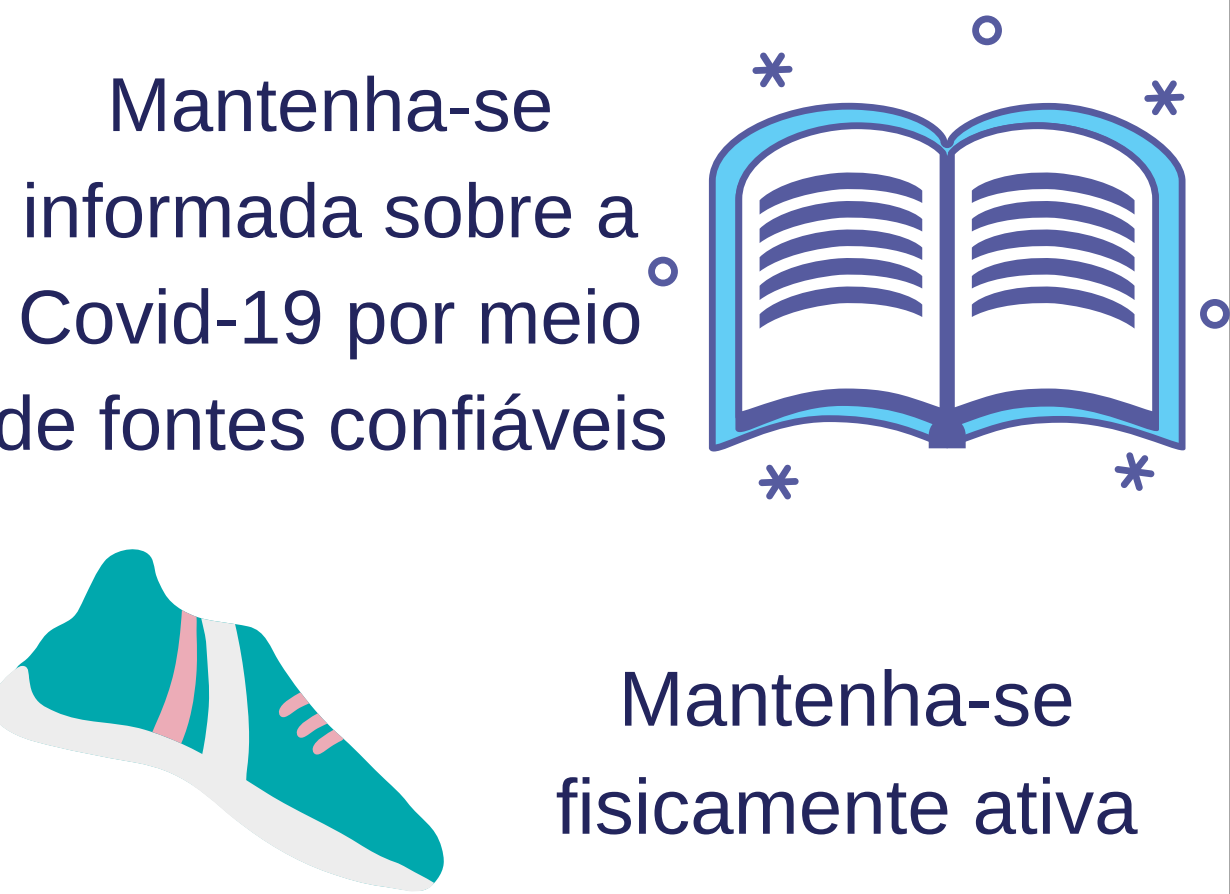

\section{Mantenha-se}

fisicamente ativa

Discuta suas dúvidas

e preocupações com ح

o médico responsável

pelo seu pré- natal 


\title{
EM CASO DE DÚVIDAS, CONVERSE COM OS
}

PROFISSIONAIS QUE ATENDEM SUA COMUNIDADE

\author{
E BUSQUE INFORMACÕES \\ NO SITE DO MINISTÉRIO DA SAÚDE \\ WWW.CORONAVIRUS.SAUDE.GOV.BR \\ OU \\ LIGUE 136 - DISQUE SAÚDE \\ OU
}

LIGUE 08002004300 - LINHA OFICIAL DA UEPG PARA DÚVIDAS SOBRE O CORONAVÍRUS 


\section{Gestação e}

medicamentos

\section{NÃO SE AUTOMEDIQUE!}

Alguns medicamentos passam para o leite materno e podem causar efeitos colaterais no bebê.

Qualquer dúvida sobre medicamentos, consulte seu médico ou farmacêutico 


\section{Medicamentos como}

\section{corticosteroides}

(exemplos: dexametasona, betametasona, prednisolona) e antiinflamatórios não esteroidais - AINEs + (exemplos: ibuprofeno, cetoprofeno, diclofenaco, nimesulida) podem ser altamente prejudiciais às grávidas! 
Se você tiver alguma doença crônica

(hipertensão, diabetes, artrite, entre outras)

\title{
NÃO ABANDONE 0 TRATAMENTO
}

\author{
pois a doença pode \\ agravar-se!
}




\section{Puérperas}

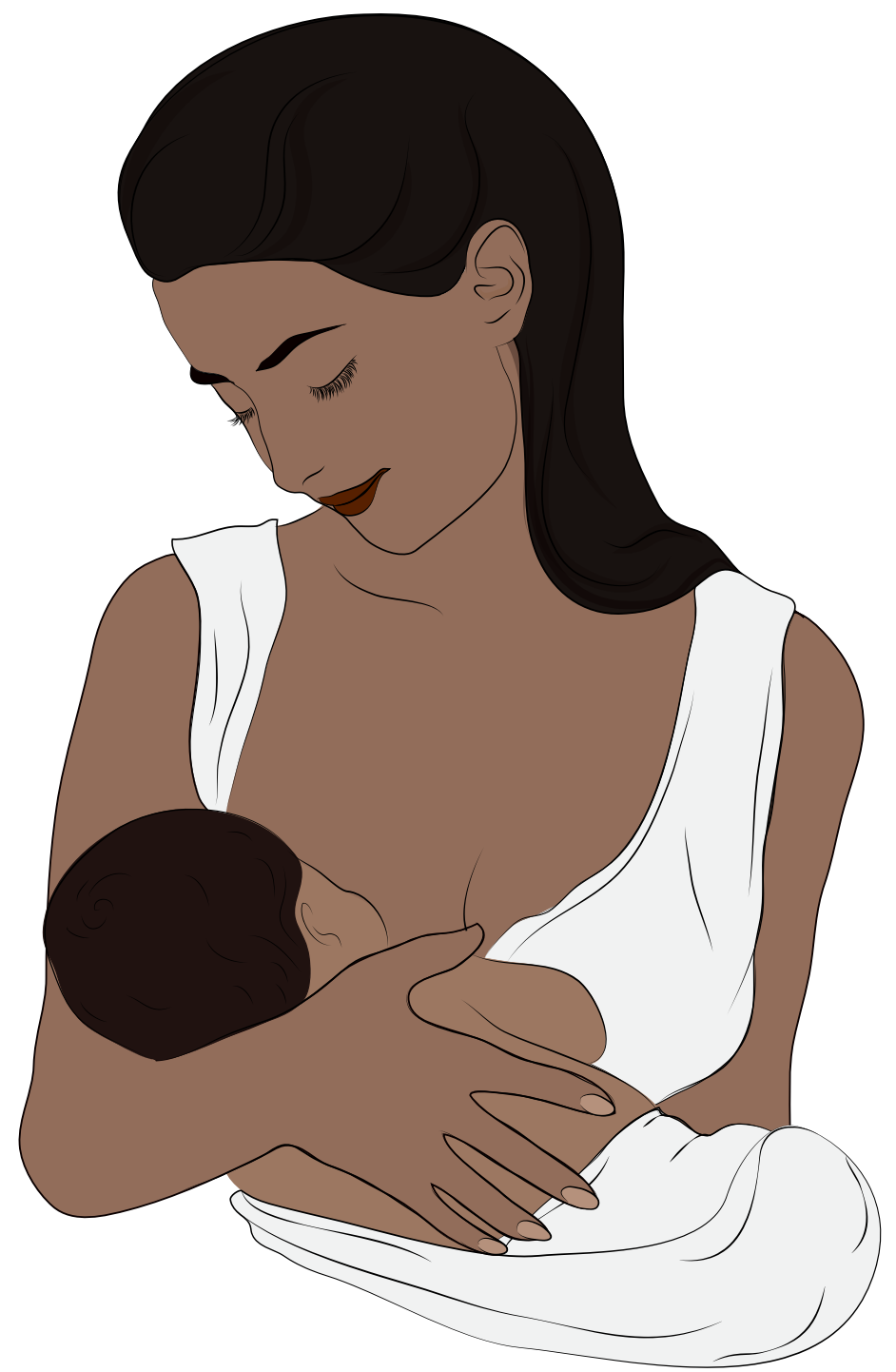




\section{O QUE É PUERPÉRIO?}

\section{É o intervalo que ocorre depois do nascimento}

\section{DURA CERCA DE 4 A 6}

\section{SEMANAS}

As alterações anatômicas e fisiológicas maternas induzidas pela gravidez retornam a seu estado original 


\section{Puerpério e Covid-19}

\section{Dados disponíveis até o}

momento não mostram que a infecção tem mais

risco de gravidade se ocorrer na gravidez ou após o parto

\section{OS RISCOS}

\section{SÃO OS MESMOS DAS}

\section{MULHERES NÃO GRÁVIDAS}

\section{DA MESMA IDADE}




\section{Riscos ao \\ recém-nascido \\ Há poucos relatos na}

literatura médica a respeito

de infecção em crianças

\section{MAS,}

\section{SABE-SE QUE O RISCO DE MORTE}

\section{É BAIXO NESSA FAIXA DE IDADE}




\section{O ALEITAMENTO MATERNO}

\section{DEVE SER MANTIDO E}

\section{INCENTIVADO}

Mesmo com

suspeita de

\section{Covid-19}

\section{máscara}

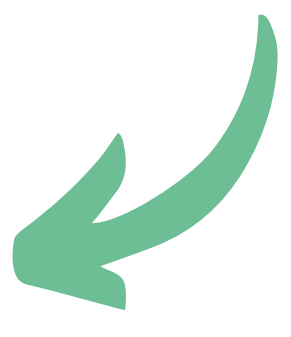




\section{As mulheres com}

suspeita de Covid-19 que estejam amamentando não devem parar sem uma avaliação de risco-benefício

Em estudos limitados, a Covid-19 não foi detectada no leite

\section{materno}




\section{O LEITE MATERNO OFERECE}

\section{PROTEÇÃO CONTRA MUITAS}

\section{DOENÇAS, INCLUSIVE}

CONTRA VÍRUS, EÉ A

MELHOR FONTE DE

NUTRIÇÃO PARA A

MAIORIA DOS BEBÊS 


\section{RECOMENDAÇÕES \\ PARA TODA A \\ POPULAÇÃO}

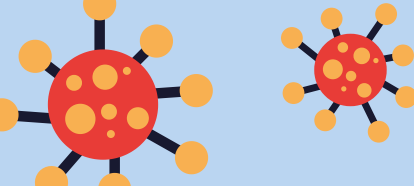

$$
\text { ró }
$$




\section{Lave as mãos com água $\mathrm{e}$}

sabão ou passe álcool em gel
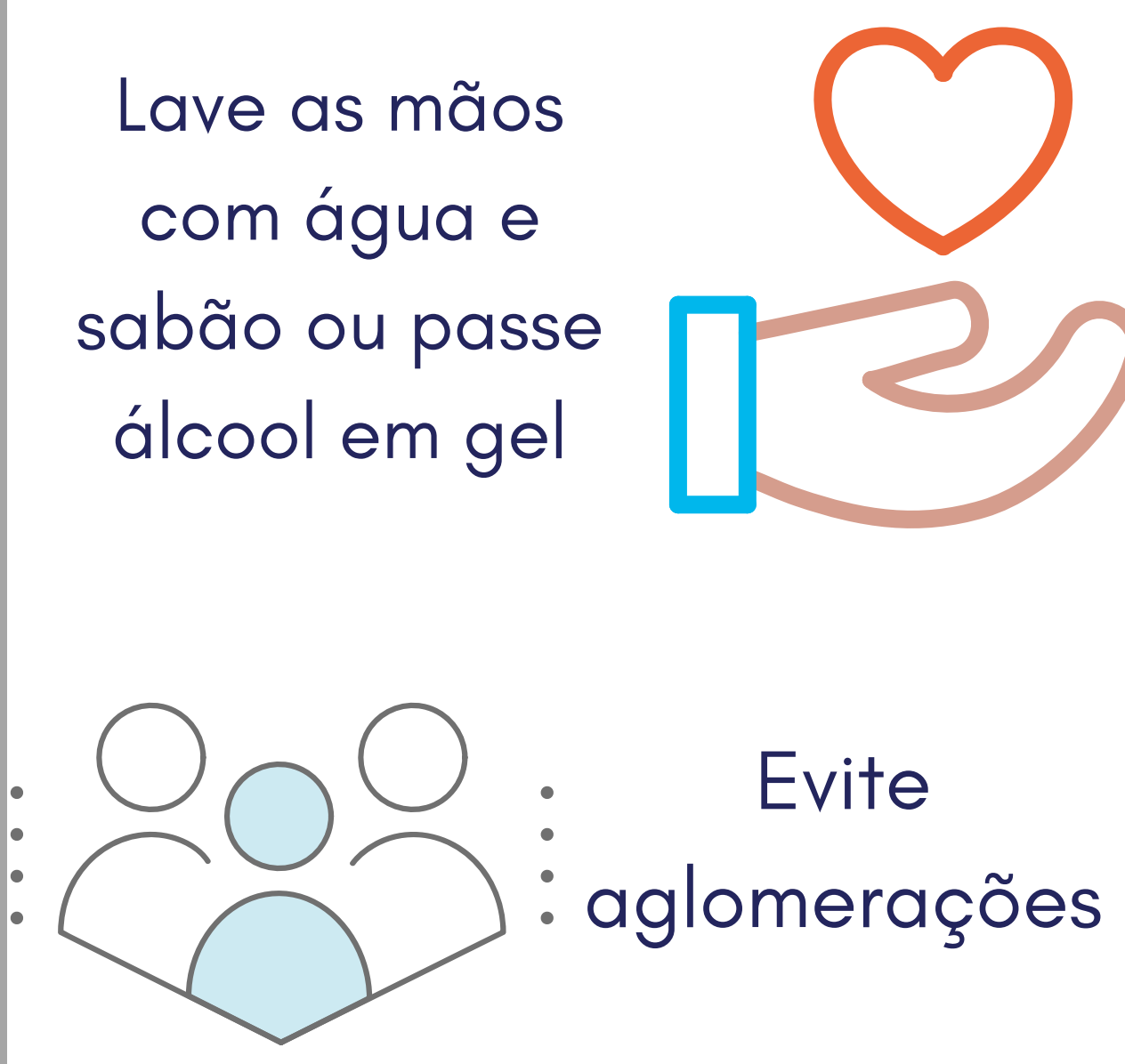

Evite tocar no

rosto, no nariz e nos olhos

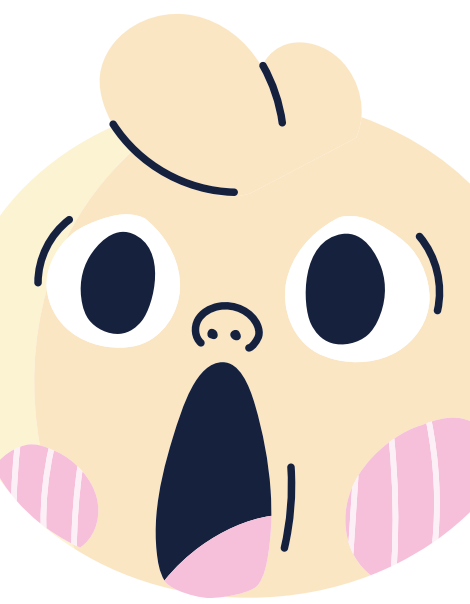




\section{Evite contato}

com doentes, especialmente pessoas com tosse

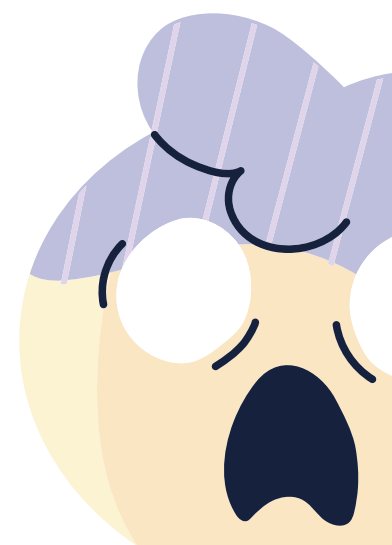

Se precisar sair, use máscara

\section{Fique em casa!}

ISOLAMENTO SOCIAL É IMPORTANTE 


\section{Referências}

BRASIL. Ministério da Saúde. Secretaria de Atenção à Saúde. Departamento de Ações Programáticas e Estratégicas.

Amamentação e uso de medicamentos e outras substâncias. 2. ed. Brasília, 2010. (Série A. Normas e Manuais Técnicos).

Disponível em: ttp://bvsms.saude.gov.br/bvs/publicacoes/amame ntacao_uso_medicamentos_2ed.pdf. Acesso em: 16 abr. 2020.

BRASIL. Ministério da Saúde. Secretaria de Atenção Especializada à Saúde. Departamento de Atenção Hospitalar, Domiciliar e de Urgência. Protocolo de Manejo Clínico da Covid-19 na Atenção Especializada. Brasília, 2020. Disponível em: https://portalarquivos.saude.gov.br/images/pdf/2020/April/14/ Protocolo-de-Manejo-Cl--nico-para-o-Covid-19.pdf. Acesso em: 16 abr. 2020.

BRASIL. Ministério da Saúde. Secretaria de Ciência, Tecnologia, Inovação e Insumos Estratégicos em Saúde. Departamento de Gestão e Incorporação de Tecnologias e Inovação em Saúde. Diretrizes para diagnóstico e tratamento da Covid-19. Brasília, 8 abr. 2020. Disponível em: https://portalarquivos.saude. gov.br/images/pdf/2020/April/13/Diretrizes-COVID-13-4.pdf. Acesso em: 16 abr. 2020.

CDC - CENTERS FOR DISEASE CONTROL AND PREVENTION. Pregnancy and Breastfeeding. 15 Apr. 2020. Disponível em: https://www.cdc.gov/coronavirus/2019-ncov/needextraprecautions/pregnancy-breastfeeding.html. Acesso em: 16 abr. 2020. 
$\mathrm{CHEN}, \mathrm{H}$. et al. Clinical Characteristics and Intrauterine Vertical Transmission Potential of Covid-19 Infection in Nine Pregnant Women: a Retrospective Review of Medical Records. Lancet, v. 395, p. 809-815, Feb. 2020. Disponível em: ttps://www.thelancet.com/pdf/journals/lancet/PIIS01406736(20)30360-3.pdf. Acesso em: 16 abr. 2020.

FEBRASGO - FEDERAÇÃO BRASILEIRA DAS ASSOCIAÇÕES DE GINECOLOGIA E OBSTETRÍCIA. Infecção pelo coronavírus SARS-CoV-2 em obstetrícia: enfrentando o desconhecido! 18 mar. 2020. Disponível em: $<$ https://www.febrasgo.org.br/en/ex-presidentes/item/948infeccao-pelo-coronavirus-sars-cov-2-em-obstetricia-enfrentandoodesconhecido>. Acesso: 16 abr. 2020.

LIANG, H.; ACHARYA, G. Novel Corona Virus Disease (Covid19) in Pregnancy: What Clinical Recommendations to Follow? Acta Obstet Gynecol Scand, v. 99, p. 439-442, 2020. Disponível em: https://obgyn.onlinelibrary.wiley.com/ doi/pdf/10.1111/aogs.13836. Acesso em: 16 abr. 2020.

PARANÁ. Secretaria da Saúde. Sesa já publicou 12 Notas Orientativas para profissionais da área com recomendações sobre o coronavírus. 30 mar. 2020. Disponível em:

http://www.saude.pr.gov.br/modules/noticias larticle.php?storyid=7239. Acesso em: 16 abr. 2020.

RASMUSSEN S.A. et al. Coronavirus Disease 2019 (Covid-19) and Pregnancy: What Obstetricians Need to Know. American Journal of Obstetrics \& Gynecology, 24 Feb. 2020. Disponível em: https://www.ajog.org/article/S0002-9378(20)30197-6/fulltext. Acesso em: 16 abr. 2020 